\title{
Strates
}

STRATES Matériaux pour la recherche en sciences sociales

$12 \mid 2006$

Nouvelles tensions impériales et recompositions en Europe centrale, orientale, et CEI

\section{De quelques représentations de la nation dans le théâtre biélorussien}

Virginie SYMANIEC

\section{(2) OpenEdition}

\section{Journals}

Édition électronique

URL : http://journals.openedition.org/strates/1922

DOI : $10.4000 /$ strates.1922

ISSN : $1777-5442$

Éditeur

Laboratoire Ladyss

Édition imprimée

Date de publication : 31 décembre 2006

ISSN : 0768-8067

Référence électronique

Virginie SYMANIEC, «De quelques représentations de la nation dans le théâtre biélorussien », Strates

[En ligne], 12 | 2006, mis en ligne le 19 juillet 2007, consulté le 08 septembre 2020. URL : http://

journals.openedition.org/strates/1922 ; DOI : https://doi.org/10.4000/strates.1922

Ce document a été généré automatiquement le 8 septembre 2020.

Tous droits réservés 


\title{
De quelques représentations de la nation dans le théâtre biélorussien
}

\author{
Virginie SYMANIEC
}

1 La Biélorussie, ancienne république soviétique, proclame son indépendance en 1991. Sous l'égide du gouvernement de Stanislas Chouchkiévitch, ce pays, situé à l'Est de la Pologne, à l'Ouest de la Russie, au Sud des pays Baltes et au Nord de l'Ukraine, peuplé à l'époque d'un peu plus de dix millions d'habitants et fortement touché par les conséquences de la catastrophe de Tchernobyl, tente d'abord de se tourner vers l'Europe, mais sans succès. En 1994, Aleksandr Loukachenko est plébiscité à plus de $80 \%$ des suffrages comme premier président de la république sur la base d'un programme anti-corruption.

2 Progressivement, il met en place un régime autoritaire fondé sur une idéologie populiste dont certains aspects doivent beaucoup au panslavisme, et d'autres à une nostalgie désormais cultivée par lui pour le régime soviétique. Aleksandr Loukachenko prône notamment le rapprochement de son pays avec la Russie, non sans susciter une forte opposition chez ceux qui voient, dans sa politique, une remise en question de la souveraineté et de l'indépendance du pays. La seconde moitié des années 1990 aura donc été marquée, en Biélorussie, par un renouveau de la violence en politique, certains événements rappelant la guerre qui opposa, au début du $\mathrm{xx}^{\mathrm{e}}$ siècle, les partisans d'un nationalisme de type traditionnel aux défenseurs d'un patriotisme d'État biélorussien et soviétique. L'entrecroisement de conceptions nationalistes et populistes reste notamment visible au sein des textes dramatiques biélorussianophones de l'époque.

3 Le théâtre apparaît comme un bon baromètre des diverses formes de représentations qu'une société se donne à elle-même. Il semble être aussi un bon instrument de politisation de la langue qu'il donne à voir et à entendre. Phénomène symptomatique, les premiers textes dramatiques strictement biélorussianophones succèdent à l'apparition de partis politiques biélorussianisants et furent majoritairement écrits par des auteurs dramatiques, militants du mouvement de "renaissance nationale" (Adradjennie) de la fin du XIX et du début du Xxe siècle. 
4 Aussi nous semble-t-il pertinent d'étudier les représentations de la nation et de l'identité des Biélorussiens dans les textes dramatiques de l'époque, sachant qu'une des caractéristiques de cette dramaturgie au service de la cause nationale est sa politisation croissante.

5 Parmi les textes de la cinquantaine d'auteurs dramatiques biélorussianophones recensés sur cette période ${ }^{1}$, plusieurs répétitions structurelles peuvent servir d'appui à notre réflexion. Loin de les étudier toutes, nous nous concentrerons sur la plus pertinente, à savoir l'évolution d'usage des motifs des fiançailles et du mariage dans trois adaptations de Paùlinka de Ianka Koupala² (1913-1996).

Du mariage

6 L'une des principales répétitions structurelles de la dramaturgie biélorussianophone pré-révolutionnaire est l'utilisation du thème du mariage souvent conjugué à celui de la fête. Une jeune fille métaphore de la nation, généralement pure, dont le tempérament et la langue soulignent la biélorussianité, aime un jeune homme local qui, à son contact, devient également l'enjeu de diverses interprétations symboliques. Les jeunes gens à marier n'ont toutefois pas le même profil selon que les noces sont consommées, contrariées ou rompues. Selon les cas, la problématique du mariage peut autant servir de support aux idées d'union et d'harmonie nationales que devenir emblématique d'une révolution désirée ou exécrée. Le traitement de ce thème se laisse donc facilement politiser. Il varie également en fonction des catégories de nation et de classe que les jeunes gens à marier permettent de mettre en présence, d'associer ou d'opposer. Il peut, en effet, arriver que les jeunes amoureux soient de même nationalité, mais de classes sociales différentes, schéma qui n'est à première vue guère original puisqu'on le retrouve de manière récurrente dans tout le théâtre occidental sans pour autant que soit posée la question de ses traitements nationalistes. Seule la référence au contexte politique et social de la Biélorussie du début du siècle permet de creuser cette possibilité d'interprétation.

7 Paùlinka de I. Koupala est certainement l'une des pièces les plus pertinentes à étudier de ce point de vue. L'héroïne du même nom, fille de Stiapan, un aristocrate de province, est amoureuse de Iakim, révolutionnaire d'origine paysanne. L'originalité de cette pièce réside en ce que les noces ne peuvent pas être consommées du fait de l'arrestation de Iakim, dénoncé aux autorités par le père de la jeune fille. Il peut aussi arriver que les noces soient contrariées alors que les deux amoureux sont de même nationalité et de même classe sociale. C'est par exemple le cas de Hapka, paysanne biélorussienne et de Biaz'rodny (sans famille), paysan biélorussien dans Biaz'rodny d'Ouladzislaù Haloubok ${ }^{3}$, où la séparation tragique des amoureux est autant due à l'autoritarisme paternel qu'aux événements extérieurs, en l'occurrence, la Première Guerre mondiale. Un troisième schéma met en présence deux amoureux qui ne sont ni de même nationalité, ni de même rang social. Dans Ce n'est pas la raison qui fatigue mais le cour 4 , Kas'ian Viasioly ajoute même une nuance. Si Nasta est fille d'un riche paysan biélorussien, Grychka est fils d'un pauvre paysan biélorussien. Mais la nationalité de ce dernier est considérée comme pervertie du fait de son statut social: enrôlé dans l'armée impériale russe, il est tout au long du texte qualifié de maskal', mot péjoratif substitué à moscovite.

8 Dans la plupart des cas, le principal obstacle au mariage d'amour est le couple père/ prétendant légitime choisi par le père, qui symbolise un pouvoir autoritaire. En l'occurrence, le paternalisme est souvent traité comme métonymie du pouvoir tsariste. 
La récurrence de ce phénomène permet de nuancer les analyses des principaux historiens du théâtre biélorussien soviétique pour qui l'opposition des protagonistes renvoie presque uniquement à des conflits entre classes ou entre générations. Car il se trouve que les opposants au mariage d'amour entre deux membres de la communauté nationale, quelle que soit leur classe, situent toujours le conflit au plan culturel en introduisant l'élément clé qu'est le prétendant légitime de la jeune fille à marier. Dans la majorité des cas, ce prétendant légitime choisi par le père porte la marque de l'étranger, ce qui pose en creux les questions de la concurrence entre nationalités et de la dégradation possible, par le mélange, de la nationalité biélorussienne. Il se peut en effet que le prétendant légitime choisi par le père parle une autre langue, signe dès lors explicite qu'il est d'une nationalité distincte de celle de la jeune fille à marier. C'est par exemple le cas du personnage de Pantsiéla dans Biaz'rodny d'Haloubok qui parle russe dans un environnement où tous les autres personnages parlent biélorussien. Dans ce cas précis, la jeune héroïne biélorussienne résiste à une alliance imposée de manière autoritaire avec un représentant de la Russie.

Il arrive que le prétendant légitime soit désigné comme un membre de la communauté des Biélorussiens, mais dont la nationalité est comme pervertie par le rang social. C'est par exemple le cas du personnage de Bykoùski dans Paùlinka de I. Koupala, aristocrate biélorussien qui porte toutes les marques (exceptée la langue) de la culture polonaise. Il arrive également que les opposants soient désignés comme étrangers par leur classe. Lorsque le principal opposant est un seigneur, il n'est, pour ainsi dire, jamais montré comme intégré à la communauté biélorussienne locale supposée exclusivement paysanne. Il apparaît comme un de ses dominants autoritaire et étranger. Deux présupposés sont, dans ce cas, imbriqués : de sang aristocratique et de sang étranger (polonais ou russe), le seigneur apparaît donc toujours deux fois étranger au paysan biélorussien.

Dans la plupart des cas, les adjuvants au jeune couple sont des personnages de leur génération, mais pas toujours. Ces derniers peuvent aussi bien être extérieurs au noyau familial que faire partie de ses membres (la mère, le frère, la camarade, la marraine, plus rarement le père ou le tuteur). Le Gentilhomme à la mode de Karous' Kahaniets ${ }^{5}$ reste l'exception qui confirme la règle puisque le prétendant légitime, pour une fois, n'agit pas comme adjuvant de l'instance paternelle mais comme opposant. Dans cette pièce, le père soutient l'idée du mariage d'amour contre le mariage d'intérêt face à un prétendant dont les attitudes étrangères lui déplaisent. Dans ce cas, l'enjeu principal devient l'alliance entre personnes d'une même communauté nationale, la question du rang social des amoureux semblant couler de source puisque rang et nationalité sont présentés comme intimement liées. Le mariage d'amour finit, dans ce cas, par être consommé à la défaveur du mariage d'intérêt avec un prétendant certes d'une condition sociale supérieure mais stigmatisé comme étranger et qui, cette fois encore, fait partie de cette noblesse locale qu'on ridiculise en l'opposant à la simplicité " authentique » du bon sens paysan de la jeune fille à marier. Ce prétendant, dont la langue est truffée de polonisme dès lors qu'il parle en public, mais dont le biélorussien est parfait dès lors qu'il monologue ou qu'il fait des apartés, finit même par être dénudé en public. La langue comme signe de la nationalité est ici principalement utilisée comme masque social. L'harmonie du monde local est, dans ce cas précis, rétablie par la représentation de la fête du mariage, où la jeune fille à marier épouse un jeune homme de son village et de sa condition. 
11 L'autre exception sur laquelle nous aurons l'occasion de revenir est L'idylle de Vintsent Dounine-Martsinkievitch ${ }^{6}$. Les visées de l'héroïne s'y réalisent avec le consentement du père, mais il n'est pas démontré que le mariage entre membres d'une même classe sociale ne relève pas d'une autre forme de mariage d'intérêt, l'intérêt national correspondant cette fois à l'intérêt de condition.

Dramaturgies de l'union ou de la rupture nationales?

12 Comme dans Paùlinka de I. Koupala, il arrive que le prétendant étranger soit ridiculisé sans pour autant que l'harmonie du corps social soit retrouvée. Le mariage d'amour entre la représentante aristocratique de la nation et le représentant révolutionnaire de la paysannerie ou de la petite intelligentsia ne peut pas être consommé. Si dès les premières créations dramatiques originales en biélorussien, le thème du mariage avait affirmé sa dimension symbolique sur les scènes nationalistes, soulignant l'existence de dramaturgies de l'union nationale, I. Koupala inaugure un renversement de perspective dans sa Paùlinka, publiée et jouée pour la première fois en 1913. La symbolique du mariage s'y affirme dans toute son ambivalence, compensée par le motif de "l'union impossible ».

13 Trois thèses contemporaines se sont jusqu'à présent opposées sur cette pièce. Pour l'historien du théâtre soviétique Ouladzimir Nafiod ${ }^{7}$, Paùlinka symbolise la lutte de la jeune génération contre le vieux monde, vue au travers du prisme de la lutte des classes :

Leur principal opposant [aux amoureux, écrit Nafiod] est Stiapan Krynitsky koulak, homme grossier et despotique qui, du fait de son état d'esprit ou de ses considérations intéressées, conduit presque sa fille au suicide ${ }^{8}$.

14 Zianon Pazniak, archéologue, théâtrologue et ancien dirigeant du Front populaire de Biélorussie, conteste cette interprétation ${ }^{9}$. Pour lui, la fable ne se résume pas à une simple intrigue amoureuse au service de la lutte entre les classes ou les générations. L'enjeu de la pièce concernerait plutôt «le droit de prendre en main son destin ${ }^{10}$ ". Ajoutons qu'au-delà du strict point de vue de la jeune fille, cette dimension pourrait concerner l'idée de la nation biélorussienne tout entière. Quant à l'historien Ousikaù ${ }^{11}$, il définit la pièce comme une comédie satirique et lyrique :

un véritable poème - un chant en l'honneur du courage et de l'indépendance d'une jeune fille, qui a grandi dans le vieux monde, mais qui aspire déjà à une vie nouvelle ${ }^{12}$.

15 La relation à la Biélorussie telle que définie par le discours nationaliste est presque ici évidente. En 1927, dans son article intitulé «La dramaturgie biélorussienne ${ }^{13}$, le critique Zamotsine soulignait déjà l'ambivalence du personnage de la jeune fille qui « danse la polka avec le seigneur Adolf » et qui « joue à l'idiot ${ }^{14}$ avec lui ». Y avait-il une gène à voir le personnage métaphorique de la Biélorussie s'amuser avec ou se «jouer » $\mathrm{du}$ personnage du noble polonais dans les bras duquel ses parents tentaient de la pousser?

16 Si la pièce eut un tel retentissement, c'est qu'il est aisé d'en faire une lecture politique. Cette possibilité est confirmée par l'historien du théâtre Arsen Labovitch qui, dans Théâtre de Combat ${ }^{15}$, explique la manière dont la pièce fut reçue en Biélorussie orientale sous occupation polonaise.

Dans la mise en scène de Paùlinka, [...] nous nous sommes efforcés de montrer avant tout des gens vivants, nos contemporains. Et quand bien même les événements de la comédie ne correspondent pas à l'actualité, nous les avons traité dans une perspective contemporaine. Nous avons concentré notre attention en particulier 
sur les images de Iakim, Paùlinka, Bykoùski [...]. Ce [dernier] personnage s'est transformé en une figure générale du nationalisme polonais, qui utilise le soutien des autorités, mais pas l'aide des simples travailleurs ${ }^{16}$. pourtant évolutif. Dans Paùlinka, seule l'héroïne peut être perçue comme allégorique d'une Biélorussie, qui, pour prendre en main son destin, doit accomplir une révolution intérieure à laquelle la volonté du père, représentation de l'autorité, fait obstacle. Dans Le nid détruit ${ }^{18}$, le personnage féminin principal s'égare au point de perdre la raison et de suivre, en dernier recours, le chemin qu'un Inconnu lui trace. L'allégorie nationale se lit, mais d'une autre manière, dans le tableau que I. Koupala dresse de la famille biélorussienne éclatée, dont les membres sont contraints, pour finir, d'emprunter des chemins différents, idée que l'auteur développera sous un angle tout à fait nouveau et dénué de symbolisme dans sa dernière pièce, Les Gens d'ici ${ }^{19}$.

Z. Pazniak n'a donc pas tort lorsqu'il considère que l'enjeu de Paùlinka est celui d'une prise d'indépendance, laquelle, rapportée au contexte d'écriture de la pièce, peut être déclinée du plan individuel et strictement familial au plan collectif d'une société toute entière. En effet, l'aspiration de la jeune héroïne rapportée au contexte de sa famille bien particulière a valeur métonymique. La relation de Paùlinka à la nation est renforcée par le fait que ses caractéristiques correspondent à celles que le discours nationaliste utilise pour décrire cette notion: sentimentale et romanesque, enjouée, mais pouvant sombrer dans la mélancolie, simple, chaste, croyante, jeune et vive, parlant un biélorussien empreint d'expressions populaires, au fait des traditions, chantante et musicale, elle est aussi la seule femme de la pièce à qui les hommes, hormis Stiapan, symbole de l'autorité, accordent une certaine attention. La relation de la jeune héroïne à la nation devient encore plus prégnante lorsqu'apparaît le second destinataire du sujet, Adolf Bykoùski, comme si les éléments de la symbolique biélorussienne ne pouvaient être définie que par comparaison avec des éléments symboliques étrangers qu'on en profite pour moquer.

20 La thèse de Zianon Pazniak reste toutefois incomplète, car l'aspiration de la jeune fille rapportée au contexte de cette petite famille aristocratique biélorussienne de province peut aussi être interprétée comme une revendication révolutionnaire. Par le truchement du discours rapporté, la jeune fille est chargée par l'auteur de dévoiler les propos et les pensées du progressiste Iakim : «Nous, dit-il [Iakim], ne sommes pas des enfants - nous avons notre intelligence et nous pouvons nous diriger ", rapporte Paùlinka dès la première scène. Selon le révolutionnaire Iakim, le dénouement de l'intrigue dépend également de la nationale Paùlinka: «Toi seule, dit-il [Iakim], Paùlinka, tu dois t'en persuader. Car autrement, dit-il, nous périrons, comme des musaraignes... », poursuit la jeune fille. Tout au long de la pièce, la représentante allégorique de la nation contribue à diffuser le discours révolutionnaire et la critique sociale. Le procédé du discours rapporté permet donc à l'auteur de faire entrer son 
héroïne en politique par « imitation ». Au premier degré, la femme nationale devient le porte parole d'idéaux formulés par les hommes, mais sans pour autant que son rôle de «passeuse » d'idées lui confère une quelconque possibilité d'avoir réellement prise sur l'action.

21 En effet, la volonté de ce personnage qui n'a aucun pouvoir a finalement peu de poids. Paùlinka finit par être entièrement dépassée par l'action. Elle est même la seule à ne pas comprendre ce qui lui arrive et à se retrouver devant le fait accompli de sa propre tragédie. Iakim, qu'on a vu apparaître une seule fois, est arrêté sur dénonciation du père de la jeune fille-nation à qui vient d'être déniée toute possibilité de prendre en main son destin sur "le nouveau chemin du mariage». Le rideau se referme sur la panique provoquée par l'évanouissement de cette dernière et ce final en dit peut-être plus long sur la croyance de I. Koupala dans le succès de la « révolution nationale » que tous les discours et les prises de positions politiques " positives » que les analystes lui ont attribué par la suite.

Du nationalisme révolutionnaire au patriotisme

$\mathrm{Au}$ nationalisme révolutionnaire koupalien contrarié répond la dramaturgie patriotique légitimiste de Frantsichak Aliakhnovitch qui fiance Paùlinka. Tandis que chez I. Koupala, l'allégorie nationale ne cessait de croiser le rêve révolutionnaire, Paùlinka fiancée ${ }^{20}$ est un cas typique où le mariage d'amour réalisé opère en contradiction avec l'idée de révolution.

Il se trouve que Frantsichak Aliakhnovitch fiance Paùlinka en 1921 sous occupation polonaise tandis qu'il dirige la troupe biélorussienne du Théâtre national. Accusé de plagiat, sa pièce est néanmoins autorisée alors que l'original koupalien est retiré de l'affiche, ce qui démontrerait que les autorités d'occupation, elles, ne se trompaient pas sur le sens symbolique et politique de la pièce. Plusieurs critiques ont toutefois été formulées à l'encontre de la proposition d'Aliakhnovitch. Dans son Théâtre biélorussien ${ }^{21}$, Nafiod compare les deux Paùlinka, défendant la thèse que l'adaptation du «bourgeoisnationaliste » Aliakhnovitch visait à éteindre la révolte sociale de l'héroïne - on reverra en quoi cette interprétation n'est pas tout à fait fausse. Dans Aliakhnovitch - Activités théâtrales et politiques ${ }^{22}$, l'historien du théâtre radicalise encore son accusation. Il s'appuye sur une citation de l'écrivain Ou. Karpaù pour condamner le fait qu'Aliakhnovitch aurait souhaité ajouter un troisième acte au texte original :

Grâce à cette falsification, écrivait Karpaù en $1943^{23}$, l'orientation socio-politique de la pièce fut réduite à néant et l'histoire de l'amour tragique entre Paùlinka et [Iakim] Saroka prit la tournure candide d'une action joyeuse au final heureux - une idylle qui a rendu Koupala furieux et qui le poussa à protester avec fermeté.

Or, comme l'explique très justement l'historien du théâtre Sabaleùski dans Biélorussie théâtrale ${ }^{24}$, Aliakhnovitch sous-titrait et dédicaçait son troisième acte: Offrande à Koupala, et aucun document ne prouve que ce dernier se soit insurgé contre cette adresse. Pour Esakoù, qui penche plutôt du côté de Nafiod, Aliakhnovitch aurait plus simplement décidé de reprendre Paùlinka a son compte. "Dans quel esprit cela fut-il fait, demande t-il ? Sans doute avec l'idée d'altérer le sens de l'œuvre koupalienne ${ }^{25}$. Aliakhnovitch laisse pourtant une trace de sa propre position sur la question dans son histoire du Théâtre biélorussien ${ }^{26}$, où il semble considérer que la Paùlinka koupalienne n'est pas artistiquement aboutie; que son intrigue, trop simple, pêche par défauts dramaturgiques et que ses personnages sont incomplets du point de vue de leur construction psychologique. 
Publiée à Vilnia en 1921, Paùlinka fiancée se présente comme une pièce en un acte avec chansons et danses. Affirmer qu'il s'agirait d'une suite à la Paùlinka koupalienne relève de l'interprétation car dans cette publication, l'auteur ne la présente pas comme un "troisième acte ». Aliakhnovitch ajoute un personnage à la distribution d'origine : Adél'ka, camarade de Paùlinka, 20 ans. Le décor ne varie pas, mais Aliakhnovitch contextualise l'action dans un temps historique et non pas, comme le faisait Koupala, dans le temps symbolique de l'automne ${ }^{27}$. Chez Aliakhnovitch, «La scène se passe au cours des dernières années avant la guerre $»^{28}$, ce qui ne prédestine pas du tout le final de la même manière. Le temps aurait donc passé... Paùlinka s'est réveillée de son évanouissement et Iakim est toujours en prison.

Contrairement à Koupala, Aliakhnovitch refuse d'emblée de situer sa pièce dans une perspective révolutionnaire, ce que souligne constamment l'attitude de la mère de la jeune fille, Al'jbieta. Si chez Koupala, cette dernière bien que dénuée de pouvoir faisait partie des adjuvants de la jeune fille, l'Al'jbiéta d'Aliakhnovitch acquiert la position d'adjuvante à l'autorité paternelle. Paùlinka semble, quant à elle, avoir perdu toute fraîcheur et toute capacité de se moquer de la dite autorité. Présentée dans une attitude presque soumise, elle semble accepter son sort et ne se révolte ni contre la situation, ni contre la décision paternelle. Elle se demande simplement pourquoi Iakim a été arrêté, lui qui voulait le bien du peuple. Al'jbieta a pour réponse toute prête qu'ils « disent tous vouloir le bien du peuple» et qu'il «n'y a pas de fumée sans feu». Si Paùlinka amoureuse commence par partager la mélancolie de la Paùlinka koupalienne, elle est désormais totalement dépourvue du désir de prendre en main son destin. Elle n'a plus de révolution intérieure à accomplir, mais doit, selon les conseils de sa mère, s'en remettre plutôt à Dieu. Cette dimension est encore soulignée dans la seconde scène où une fois seule, le monologue de la jeune fille prend la forme d'une supplique adressée à Dieu, avant de se transformer en chant : "Calme toi, petit cœur, ne tremble pas ${ }^{29}$ ".

Le premier coup de théâtre qu'utilise Aliakhnovitch pour débloquer la situation ne dément pas ce changement radical de perspective. Adèl'ka, amie de Paùlinka, entre à la scène 3 pour annoncer que Iakim a jeté un billet par la fenêtre de sa cellule. Il est trouvé par des enfants qui le lui ont apporté. Cette fois, c'est la camarade de Paùlinka qui, par le truchement du discours rapporté, devient porte-parole des propos de Iakim. Ces derniers n'ont d'ailleurs plus rien de révolutionnaire ou de subversif: la police a commencé par vérifier tous ses livres, mais tous portaient la mention «Autorisé par la censure ». Comme rien ne fut trouvé chez lui de "non autorisé », on l'a emprisonné pour lui faire peur. Comme il a dit toute la vérité, il doit être libéré le jour même.

Iakim paraît à la scène 8 : «Me voilà - comme avant ${ }^{30}$ ». Mais ses retrouvailles avec Paùlinka sont interrompues par l'entrée de Stiapan, suivi d'Al'jbiéta. Stiapan est d'abord furieux de voir Iakim dans sa propre maison en train d'embrasser sa fille. Iakim parvient toutefois à le convaincre de l'écouter. Son long monologue permet de transformer le révolutionnaire koupalien en patriote adjuvant du pouvoir légitime. L'hymne à la nation, latent chez Koupala, est ouvertement hymne à la patrie chez Aliakhnovitch. L'antagonisme entre les classes qui ne trouvait aucune solution chez Koupala doit désormais être résolu au profit de la solidarité entre patriotes. En témoigne le discours de Iakim au père de la jeune fille :

Vous dites, seigneur Stiapan, que vous êtes un noble et moi un paysan... Mon bon monsieur! Quelle différence réelle y a-t-il entre nous? Sur vos papiers, il est écrit une chose et sur les miens, une autre? Les papiers n'ont rien à y voir !... Est-ce que 
je ne suis pas, comme vous, le même fils de ma patrie, la Biélorussie? Est-ce que nous nous différencions par la langue, par nos cœurs, ou par nos amours ${ }^{31}$ ?

finit d'inverser le cours de l'histoire koupalienne : Bykoùski, le premier prétendant de
Paùlinka, vient d'être arrêté. Signant un billet à ordre, il aurait par mégarde écrit un
nom étranger en place de son nom propre. Al'jbiéta de s'étonner qu'on puisse maintenant arrêter des gens pour si peu de choses ! Mais Prantsis rassure l'assemblée : on dit qu'il sera bientôt libéré. Tout est donc bien qui finit bien. Aux doutes et aux interrogations «pessimistes» nationalistes révolutionnaires koupaliennes, Aliakhnovitch avait répondu par la représentation « optimiste » d'un nationalisme de la légitimité politique, un patriotisme, où l'idée de mariage et de retour à l'ordre n'est plus contrariée.

Du patriotisme d'Aliakhnovitch au patriotisme soviétique

Cherchant à condamner les partis pris du metteur en scène Florian Jdanovitch, l'historien Esakoù nous livre quelques informations intéressantes sur la manière dont la pièce fut jouée par «La Première Société Biélorussienne du Drame et de la Comédie » dès 1917. Selon lui, la mise en scène de Jdanovitch faisait fausse route car elle détournait l'attention des spectateurs du caractère social de la pièce. Iakim et Paùlinka n'étaient pas tant considérés comme les personnages centraux de l'intrigue que la noblesse, ce qui renforçait «les moments de vaudeville » au détriment du discours social $^{32}$. Toujours selon Esakoù, I. Koupala serait lui-même intervenu pour réaffirmer que le thème principal de l'œuvre n'était pas la noblesse mais « la vie, la lutte du peuple biélorussien $»^{33}$. Pourtant, lorsque qu'en 1927, I. Dvartchanine publie une version écourtée et destinée aux écoliers de la pièce dans son second volume de La chrestomathie de la nouvelle littérature biélorussienne ${ }^{34}$, cette dernière porte encore le sous-titre Scènes de la vie de la noblesse en deux actes que même la réédition de 1992 n'osa pas reprendre. À la fin des années 1920, le premier Théâtre Biélorussien d'État (Belarouski Dziarjaùny Teatr-1, BDT-1) aurait finalement abandonné la mise en scène de Jdanovitch. L'auteur dramatique, metteur en scène et directeur de théâtre Eùstsihnieù Mirovitch aurait alors proposé une nouvelle rédaction de la pièce, resserrant encore les relations entre Iakim et Paùlinka.

Nous ne faisons ici qu'effleurer une des problématiques centrales de l'histoire du répertoire biélorussianophone: l'adaptation et la réécriture des textes dramatiques pour raisons idéologiques d'État.

34 Aucune de ces propositions ne pouvaient toutefois convenir aux autorités soviétiques qui élaborèrent de nouvelles grilles de lecture dramaturgiques. Elle soutinrent une nouvelle proposition, qui se concluait également par un happy end, mais qui permettait de sceller l'alliance des personnages symboliques de la révolution et de la nation. En 1943, une "nouvelle étape commence dans la vie du théâtre biélorussien » sous 
l'impulsion du metteur en scène Leù Litvinaù qui "s'est donné pour but» de transformer Paùlinka en " une pure comédie », raccourcissant le texte pour le délester de tout ce qui ne relevait pas des critères généralement admis pour ce genre ${ }^{35}$ et de sa «morale bourgeoise». Le premier monologue de Paùlinka, "bien trop sérieux", disparaît ainsi que sa dernière réplique, connue sous le nom de "malédiction de Paùlinka » : « fauves aveugles ». L'arrestation de Iakim sur dénonciation est désormais traitée sur le mode comique. Stiapan et Poustarévitch perdent leur rang d'aristocrates et deviennent de bons paysans biélorussiens alcooliques, ce qui permet au passage de nier la problématique du conflit de classe interne à la société paysanne biélorussienne et de concentrer le tir sur le personnage de Bykoùski, aristocrate polonais.

Suite à l'indépendance de 1991, l'original koupalien avait retrouvé ses lettres de noblesse. Mais en 1996, à l'heure où le régime biélorussien dérape vers l'autoritarisme, une nouvelle première est annoncée au Théâtre National Académique d'État I. Koupala. Une dame en costume folklorique introduit la pièce, expliquant à quel point le théâtre est honoré de pouvoir réhabiliter la version de 1943 ! À la même époque, cent mille personnes, menées par quelques "Iakim contemporains » (professeurs, pédagogues, linguistes, intellectuels biélorussianophones) manifestent à Minsk et dans d'autres villes de Biélorussie contre la récente signature du premier traité d'intégration économique biélorussiano-russe qu'ils considèrent comme une atteinte portée à la souveraineté et à l'indépendance de leur pays. C'est le Printemps 96, suivi par le renouveau des répressions politiques en Biélorussie.

Printemps 2002

\section{NOTES}

1. Voir V. Symaniec, Des dramaturgies biélorussiennes à la dramaturgie biélorussienne soviétique, une tragédie de pouvoir, thèse de doctorat soutenue en 2000 , Institut d'études théâtrales de Paris III - Sorbonne nouvelle sous la direction de Martine de Rougemont, 3 volumes, $851 \mathrm{p}$.

2. Voir Dramatytchnyïa tvory [ÆEuvres dramatiques], Navouka i tekhnika, 1975, t. 6,

p. 179-236.

3. Voir Tvory [๕uvres], Mastastskaïa litaratura, Minsk, 1983, p. 150-179.

4. Vilnia, 1927, $48 \mathrm{p}$.

5. Tavarystva belaruskae shkoly, Vilnia, 1928, p. 21-32.

6. Opera ù 2-kh aktakh na belaruskaï movy [Opéra en deux actes en langue biélorussienne], 1846, 70 p., DAMLiMRB, F. 44, Klimovitch Mikhail Mikalaevitch, vop. 1, adz. Zakh. 537.

7. Voir Belarouski teatr [Le théâtre biélorussien], Akademii Navouk Belarouskaï SSR, Minsk, 1959, 169 p.

8. Ibidem, 1959, p. 77.

9. Pozniak Zenon, Problemy stanovleniïa i razvitiia belorusskogo professional'nogo teatra natchala XX v. (1900-1917 gg.) [Problèmes de la mise en place et du développement du théâtre biélorussien professionnel au début du xx e siècle (1900-1917)], Leningradskiï gosudapstvennyï institut teatra, musyki i kinematografa, 1981, 188 p. 
10. Ibidem, 1981, p 10.

11. Ousikaù Iakub, Belaruskaïa Kamedyïa [La comédie biélorussienne], Vycheïchaïa Shkola, Minsk, 1964, 208 p.

12. Il dit s'accorder avec la vision d'un certain A. Saladoùnikaù, publiée dans la Pravda

du 17 février 1955. Ibidem, 1964, p. 14.

13. Ouzvychcha, $\mathrm{n}^{\circ} 1,1927$, p. 71-82.

14. Il s'agit d'un jeu de cartes.

15. Navouka i tekhnika, Minsk, 1969, 102 p.

16. Ibidem, 1969, p. 28.

17. I. Kupala, Paemy, Dramatytchnyïa tvory [Poèmes, œuvres dramatiques],

Mast. Lit., Minsk, 1989, p. 484-493.

18. Dramatytchnyïa tvory, op. cit., p. 266-342.

19. Il est possible d'appliquer cette hypothèse à la plupart des pièces de I. Koupala, y compris au personnage de Lounianka dans Extraits d'un poème dramatique. Ibidem, p. 165-178; op. cit., 1989, p. 291-300, comme à la plupart des personnages féminins des auteurs nationalistes de l'époque. La pièce Les Gens d'ici doit toutefois faire l'objet d'une analyse particulière ; op. cit., 1989, p. 409-483.

20. Adbitka z Belarouskaha Zvony, Vilnia, 1921, 23 p.

21. Op. cit.

22. Navouka i tekhnika, Minsk, 1996, p. 49.

23. Il s'agit justement de l'année où la nouvelle mise en scène de Litvinaù transforme le final de l'original koupalien en happy end.

24. $n^{\circ} 3$ de 1992.

25. Ianka Koupala i belarouski teatr [Ianka Koupala et le théâtre biélorussien], Mastatskaïa Litaratura, Minsk, 1972, p. 55.

26. Vilnia, 1924, p. 59.

27. Comme l'écrit J. Duvignaud dans Sociologie du théâtre (Paris, Puf, 1999, p. 120) en automne, "plaisir et volupté s'éteignent. La maison enferme l'individu; il prend ses habitudes d'hiver, il se rétrécit sur lui-même ».

28. Op. cit., p. 5.

29. Op. cit., p. 7.

30. Op. cit., p. 13.

31. Op. cit., p. 17.

32. Op. cit., p. 115.

33. Op. cit., p. 116.

34. 1927, Vilnia, p. 131-149.

35. Op. cit., p. 134-152.

\section{RÉSUMÉS}

La Biélorussie est marquée par le renouveau de la violence politique depuis la seconde moitié des années 1990, opposant les partisans de l'indépendance nationale et ceux favorables à un patriotisme biélorussien de type soviétique, comme au début du siècle dernier. 
L'interpénétration des conceptions nationalistes et populistes sont visibles dans la production théâtrale du début $\mathrm{du} \mathrm{xx}^{\mathrm{e}}$ siècle écrit en biélorusse. L'auteur présente une analyse de ces pièces de théâtre comme diverses formes de représentation de la société, de la nation et de l'identité de sa langue dans une période de croissante politisation. Les textes présentés ici sont importants pour comprendre la période que vivent les Biélorussiens aujourd'hui.

Virginie Symaniec. On nation representation in the Bielorussian theater

Bielorussia is marked by the renewal of political violence since the second half of the 1990, opposing partisans of national independance and those who act in favor of soviet state Bielorrusian patriotism, as in the beginning of the century. The intercrosssing of nationalist and populist conceptions are visible inside the theater production of the early XXth century,written in Bielorussian language. The author presents an analysis of these theater plays as the diverse forms of renpresentations a society is producing on itself, with its idea of nation and identity in its own language in a period of increasing politisation. The texts presented there are, thus, important to understand the actual period Bielorussians are living to day.

\section{AUTEUR}

\section{VIRGINIE SYMANIEC}

Docteur de l'université Paris III, bart69@club-internet.fr 